www.jmscr.igmpublication.org

Impact Factor 5.84

Index Copernicus Value: 83.27

ISSN (e)-2347-176x ISSN (p) 2455-0450

crossref DOI: _https://dx.doi.org/10.18535/jmscr/v5i4.188

Journal Of Medical Science And Clinical Research

\title{
Role of Serum Magnesium in Pregnancy with Pre-Eclampsia
}

\author{
Authors \\ Dr Sophiamma Joseph, Dr Mallika Gopinath \\ Govt. MCH, TVM
}

\begin{abstract}
Background: Hypertension complicates 5-8\% of pregnancies worldwide. Hypertensive disorders of pregnancy particularly eclampsia with convulsions result in $13 \%$ of all maternal death. pre-eclampsia is the occurrence of new onset of hypertension and proteneuria after 20 weeks of gestation. MMR averages 16 per lakh live births in developed countries and 440 per lakh live births in developing regions.

Objectives

1. Analysis of risk factors of pre-eclampsia patients with that of normal pregnant women.

2. To compare the serum magnesium levels of pre-eclampsia patients with normal pregnant women.

Materials and Methods: Cross sectional study was done in a tertiary care centre. 30 pre-eclampsia patients admitted in Sree Avittom Thirunal Hospital, Thiruvananthapuram and 30 pregnant women attending Outpatient Department in the same hospital were taken. Baseline characteristics of the subjects like age, period of gestation and socio economic status were assessed. Data was collected regarding clinical features of pre-eclampsia. Magnesium was estimated by Arsenazo method by collecting $5 \mathrm{ml}$ of venous blood.

Analysis and Results: Analysis of risk factors for pre-eclampsia between pre-eclamptic women and normal women did not have any results of statistical of significance.

Analysis of clinical features of pre-eclampsia with normal pregnant women showed BMI was higher in preeclamptic women. Mean systolic and diastolic pressure were elevated among pre-eclamptic women. IUGR was more in pre-eclampsia. Magnesium levels were lowered in the first trimester of pregnancy in preeclampsia, when compared to normal pregnant women.

Conclusion: Serum magnesium was significantly lowered in the first trimister of pregnancy in women with pre-eclampsia.

Relevance of the study: In women with pre-eclampsia magnesium may be supplemented from the first trimester by magnesium rich diet and pharmacological intervention. by magnesium supplementation as tablets to reduce the incidence of pre-eclampsia.

Keywords: pre-eclampsia, Maternal Mortality rate, Serum Magnesium, IUGR.
\end{abstract}

\section{Introduction}

Hypertension complicates $5-8 \%$ of pregnancies. This forms a member of the deadly triad along with haemorrhage and infection. Of the hypertensive disorders the highest risks are associated with pre-eclampsia and eclampsia. ${ }^{1}$
Worldwide hypertensive disorders of pregnancy particularly eclampsia (convulsions) result in about $13 \%$ of all maternal deaths. Maternal mortality ratio averages at 16 per lakh live births in developed regions. In developing regions it is 440 for the same number of live births. ${ }^{2}$ 
Pre-eclampsia is the occurrence of new onset hypertension and proteinuria after 20 weeks of gestation which is specific to human pregnancy. Pre-eclampsia is connected with increased risks of cerebrovascular complications, disseminated intravascular coagulation (DIC), placental abruption, premature labour, low birth weight and even maternal and fetal death. Pre-eclampsia is a major cause of maternal and fetal morbidity and mortality. The pathophysiologic mechanism is the failure of trophoblastic invasion of spiral arteries which lead to maladaptation of maternal spiral arteries. $^{4}$

$\mathrm{Mg}$ is known to increase the prostacyclin release in endothelial cells of blood vessels. Prostacyclin is a potent vasodilator. The serum $\mathrm{Mg}^{2+}$ levels were also found to be significantly reduced in women with pre-eclampsia. Low Mg levels may potentiate contractile response of vascular smooth muscle to vasopressors. $\mathrm{Mg}$ also has a substantial beneficial effect in pre-eclampsia for the prevention and treatment of convulsions. ${ }^{5}$

Therefore, change of magnesium value during pregnancy, can modify the aetiopathology of preeclampsia. The role and status of serum magnesium in pregnant women is being discussed currently. The aim of the present study is to find out the serum levels of magnesium, in preeclampsia patients admitted in SAT hospital, Thiruvananthapuram, a tertiary care teaching hospital as there are no such previous data available. $^{6}$

\section{Objectives of the Study Primary Objective}

To find out the serum magnesium level in pregnant women with pre-eclampsia in a tertiary care centre.

\section{Secondary Objective}

To compare the serum magnesium level of preeclampsia patients with that of normal pregnant women.

\section{Review of Literature}

Hypertension is the commonest medical problem encountered in pregnancy. Mild pre-eclampsia is seen in 8 to $10 \%$ of pregnancies and severe preeclampsia in $1 \%$. This disorder was first recognized almost 2000 years ago. Celsus described women with seizure that abated with delivery. The disorder was termed 'eclampsia' meaning "flashes of light". In 1843, John Lever of Guys's Hospital in London discovered the presence of albumin by boiling the urine from pregnant women with puerperal convulsions.

According to International Society for the Study of Hypertension in Pregnancy (ISSHP) hypertension is defined as a systolic blood pressure $>140 \mathrm{~mm} \mathrm{Hg}$ or a diastolic blood pressure $>90 \mathrm{~mm} \mathrm{Hg}$ or a rise in systolic blood pressure of $30 \mathrm{~mm} \mathrm{Hg}$ or a rise in diastolic blood pressure of $15 \mathrm{~mm} \mathrm{Hg}$ at least 4 hours apart or a single diastolic blood pressure $\quad>110 \mathrm{~mm} \mathrm{Hg} .{ }^{1}$ Mean arterial pressure is calculated by the following equation:

Diastolic pressure $+1 / 3$ pulse pressure $=$ Mean arterial pressure (MAP).

A rise of $20 \mathrm{~mm} \mathrm{Hg}$ in MAP or a MAP more than 105 is significant. ${ }^{1}$ Severe hypertension and proteinuria are the diagnostic criteria of preeclampsia. ${ }^{14}$ Abnormal findings in laboratory tests of renal, hepatic and haematological function also increase certainty of pre-eclampsia. Pre monitory symptoms of eclampsia such as persistent headache and epigastric pain are also pointers to certainty. Kerala has an MMR of 66 per lac live births $^{2}$ (2010-2012). ${ }^{2}$

\section{Pathophysiology of Pre-eclampsia}

The etiology of pre-eclampsia has remained poorly understood. It is called as the disease of theories. Study by Dekker and colleagues in 1998 indicates that preeclampsia is due to genetic or immunological conflicts between the mother and the fetus. Though pathophysiology remains undefined placental ischemia is widely cited as a key factor. ${ }^{4}$ 


\section{Magnesium £ Pre-eclampsia}

Magnesium is the fourth most abundant cation in the body and the second most prevalent intracellular cation after potassium. ${ }^{96}$ In 1930s the American groups of scientists E.V. Mac Collum and D.M. Greenberg did studies on rat and showed the physiological properties and actions of Magnesium and the effects of lack of Magnesium in development, reproduction, neuromuscular transmission and hormonal balance. ${ }^{5}$ Regulation of serum Magnesium is by controlling renal reabsorption. $20 \%$ of the filtered Magnesium is reabsorbed in the cortical thick ascending limb and $5-10 \%$ in the distal convoluted tubule. Drinking water provides approximately $10 \%$ of daily Magnesium intake. Chlorophyll (and thus green vegetables are a major source of Magnesium. Other food items like unprocessed cereals, nuts and seeds are also sources of Magnesium. Milk products have very little magnesium. Processing reduces magnesium content. The recommended daily allowance is $350 \mathrm{mg} /$ day in males above 50 years old and 280 mg for females. ${ }^{6}$

Magnesium has got stimulatory effects on the production of prostacyclin and nitric oxide and alters the responses of blood vessels to vasoconstrictor agents. Magnesium is established as an essential element for fetal wellbeing. ${ }^{7}$ Systemically magnesium lowers blood pressure and alters peripheral vascular resistance. In normal pregnancy there is hypomagnesemia due to hemodilution effect of estrogen and increased demand of fetus. Increased urinary excretion of magnesium may also contribute to further decrease. $^{10}$

There is progressive decline in serum magnesium during pregnancy reaching a low level at 34 weeks and then rises up to term. Serum magnesium estimation after 2 weeks may give a clue if the woman had a chance for development of $\mathrm{PIH}^{3}$ If found to be hypomagnesemic, then magnesium supplement can be given to minimize chances of PIH.
Magnesium deficiency of ATP production and ATP dependent Sodium Potassium and Calcium pumps implicating in the development of pre eclampsia ${ }^{8}$.

\section{Materials And Methods \\ Study Design}

Cross sectional study at a tertiary care centre.

\section{Study Setting}

SAT Hospital, Thiruvananthapuram, a tertiary care teaching hospital

\section{Study Population}

\section{Cases}

Pregnant women of gestational age between 20 weeks to 38 weeks Inclusion Criteria:

\section{Singleton pregnancy}

Non diabetic, BP above $140 / 90 \mathrm{~mm} \mathrm{Hg}$ on two occasions four hours apart. Urinary protein $1+$ or more with dipstick.

\section{Exclusion Criteria}

Gestational diabetes, chronic hypertension, kidney disease, coagulation disorders, multiple pregnancy, cardiovascular disease, severe anemia, endocrine disorders.

\section{Controls}

Pregnant women of gestational age between 20 weeks to 38 weeks and same exclusion criteria with no pre-eclampsia.

\section{Sampling Technique}

Consecutive cases of pre-eclampsia patients admitted and consecutive cases of normal pregnant women attending OPD.

Sample Size Calculation

Using the formula

$$
\frac{2\left[Z\left(1-\frac{\alpha}{2}\right)+Z(1-\beta)\right]^{2} x S^{2}}{d^{2}}
$$

$=15$ each. Sample size was increased to 2 n i.e. 30 by design effect.

Mean serum magnesium among pre-eclamptic women $0.75 \mathrm{mmol}$ SD 0.08 Mean serum magnesium among normal pregnant women 0.85 mmol SD 0.09 Significance level 5\% 


\section{Data Collection}

The study was conducted after the approval of the Ethical Committee (IEC. No.01/53/2014/MCT). The details were recorded in the proforma and blood and urine samples were collected after obtaining written informed consent.

\section{Estimation of Magnesium}

Method: Arsenazo method

Sample Collection: About $5 \mathrm{ml}$ of blood was drawn under aseptic precuations from cubital vein and collected in a plain bottle. Serum was separated by centrifugation and kept at $-20^{\circ} \mathrm{C}$ until analysis was carried out. Magnesium levels were assessed by Arsenazo method using fully automated biochemical analyser.

Proforma was given to the patient regarding Base line characteristics regarding pre - eclampsia.

1. Age

2. Period of gestation

3. Gravidity

4. Social Economic status

5. Antenatal checkups carried out

The analysis of clinical features of pre-eclampsia was also carried out.

\section{Observations and Results}

The following cross sectional study was conducted among 30 pre-eclamptic women admitted and 30 normal pregnant women attending OPD in SAT Hospital. The primary objective of our study was to find the levels of magnesium, among pre-eclampsia subjects and secondary objective was to compare the levels of magnesium with that of normal pregnant women. Statistical analysis was done using SPSS software, version 20 by student's t- test for statistical significance.

Baseline Characteristics of the study population

1. Age, Period of gestation, Gravidity, Socio economic status \& periodical antenatal checkups. These parameters when analysed from the proforma there was no difference of statistical significance between the control and preeclampsia patients.
Analysis of clinical features of study population

1. Hea ache was similar in two groups

2. Oliguria was absent in both groups

3. Epigastric pain was absent in both groups

4. Foetal movements were present in all subjects

5. Dyspnoea was absent in all subjects

6. Convulsions was absent in all subjects

7. Edema was present more in preeclamptic patients, but all patients were having edema it was not considered as a positive sign.

8. Body max index

Table 1: Mean and standard deviations of body max index

\begin{tabular}{|l|c|c|c|c|}
\hline Group & Mean & SD & t & $\mathrm{p}$ \\
\hline Pre-eclampsia & 28.0 & 3.3 & \multirow{2}{*}{2.5} & \multirow{2}{*}{0.016} \\
\hline No pre-eclampsia & 26.6 & 4.1 & & \\
\hline
\end{tabular}

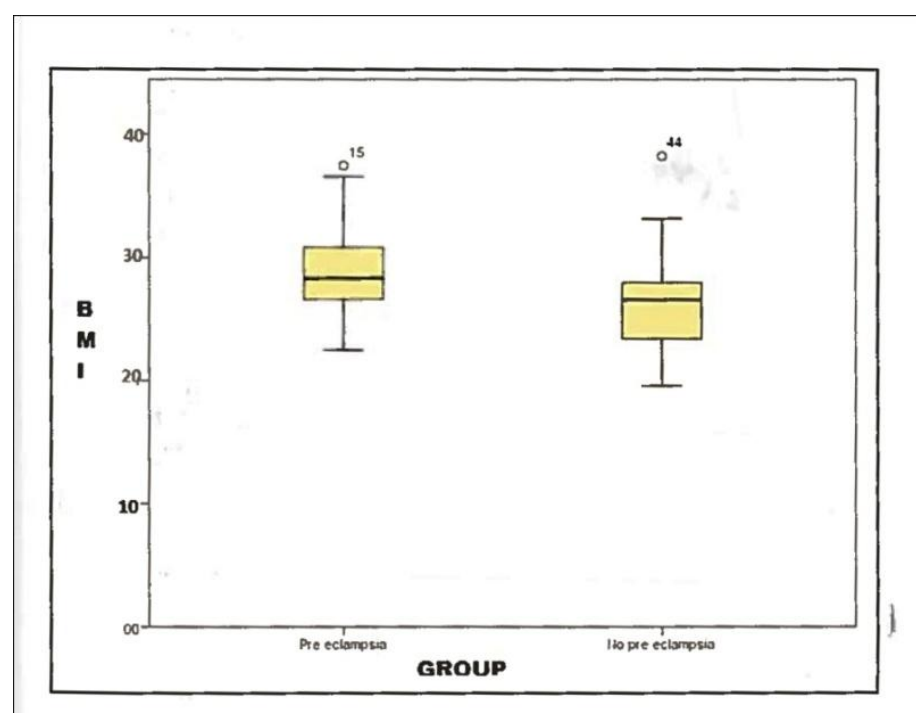

Chart 1: Distribution of subjects according to their body mass index

The mean value of BMI among pre-eclampsia patients was $28.9 \pm 3.3$ and that of normal pregnant women was $26.6 \pm 4.1$. The difference between the means is statistically significant with a $\mathrm{p}$ value of 0.016 . 


\section{JMSCR Vol||05||Issue||04||Page 20920-20927||April}

\section{Blood pressure}

Table 2: Descriptive statistics of blood pressure among study group

\begin{tabular}{|c|c|c|c|c|c|}
\hline Parameter & Group & Mean & $\begin{array}{c}\text { Standard } \\
\text { Deviation }\end{array}$ & $\mathrm{t}$ & $\mathrm{p}$ \\
\hline \multirow[t]{2}{*}{$\begin{array}{l}\text { Systolic Blood } \\
\text { pressure }\end{array}$} & Pre-eclampsia & 147.3 & 12.1 & \multirow{2}{*}{12.67} & \multirow{2}{*}{0.000} \\
\hline & Pre-eclampsia & 113.4 & 82 & & \\
\hline \multirow[t]{2}{*}{$\begin{array}{l}\text { Diastolic } \\
\text { blood pressure }\end{array}$} & Pre-eclampsia & 96.5 & 7.4 & & \multirow{2}{*}{0.000} \\
\hline & $\begin{array}{c}\text { No-pre- } \\
\text { eclampsia }\end{array}$ & 71.7 & 5.9 & 14.32 & \\
\hline
\end{tabular}

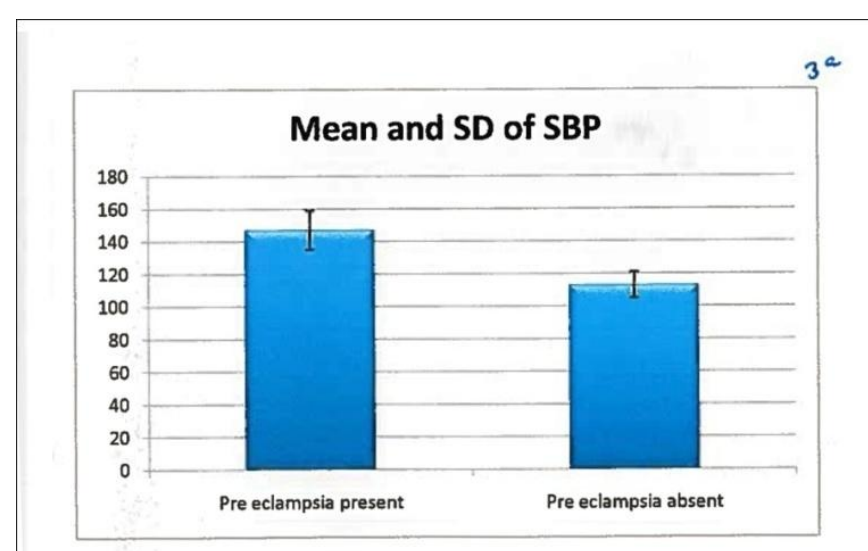

Chart: 2A. Distribution of study subjects according to systolic blood pressure.

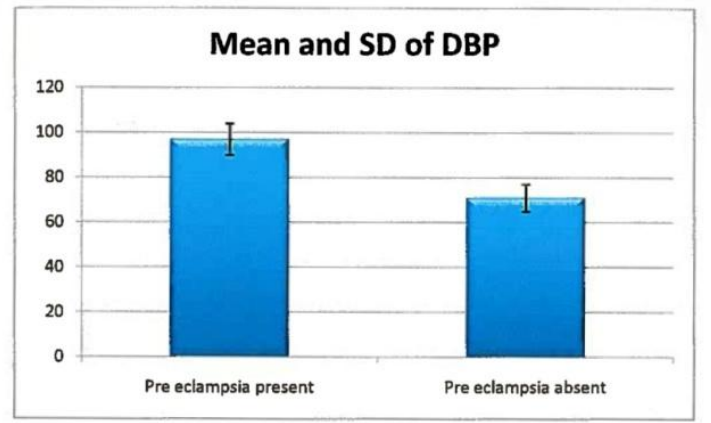

Chart: 2 B Distribution of study subjects according diastolic blood pressure

The mean systolic and diastolic blood pressure among pre elcamptic women was significantly elevated than normal pregnant women.
10. Urine protein

Table 3 Distribution of study subjects according to presence of urine protein

\begin{tabular}{|l|l|l|l|l|l|l|}
\hline \multirow{2}{*}{$\begin{array}{l}\text { Urine } \\
\text { Protein }\end{array}$} & \multicolumn{4}{|l|}{ Group } & \multicolumn{2}{|l|}{} \\
\cline { 2 - 5 } & Pre-eclampsia & \multicolumn{2}{|l|}{ No pre-eclampsia } & \multicolumn{2}{l|}{ Total } \\
\hline & Count & $\%$ & Count & $\%$ & Count & $\%$ \\
\hline Absent & 0 & 0 & 30 & 100.0 & 30 & 50.0 \\
\hline $1+$ & 29 & 96.7 & 0 & 0 & 29 & 48.3 \\
\hline $3+$ & 1 & 3.3 & 0 & 0 & 1 & 1.7 \\
\hline Total & 30 & 109.0 & 30 & 100.0 & 60 & 100.0 \\
\hline
\end{tabular}

Chi square $60 \mathrm{p}=1.000$

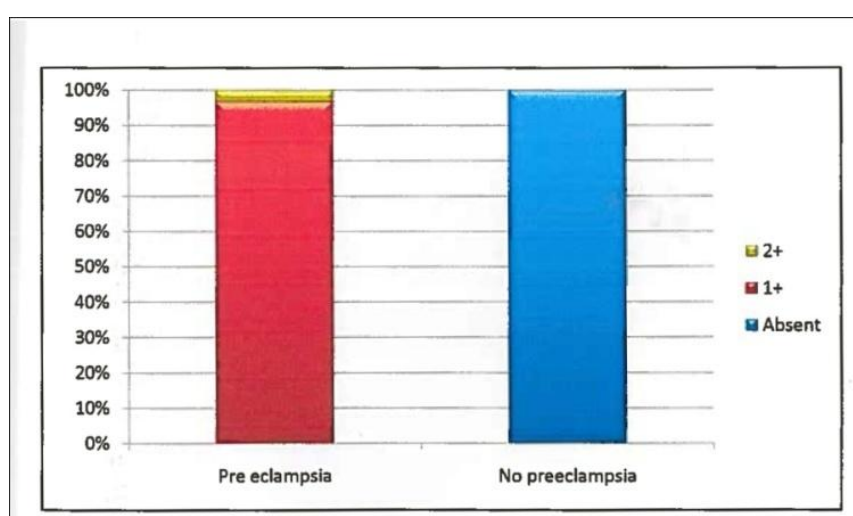

Chart 3. Distribution of study subjects according to presence of urine protein

Out of the 30 pre-eclamptic women, 29 had grade 1 protenuria and only 1 had severe proteneuria

\section{Analysis of Serum Magnesium}

Table 4. Descriptive statistics Serum Magnesium in $\mathrm{mg} \%$ in the study group

\begin{tabular}{|l|c|c|}
\hline Descriptive & Pre-eclampsia & No Pre-eclampsia \\
\hline Mean & 1.97 & 2.19 \\
\hline Median & 1.99 & 2.15 \\
\hline Std. Deviation & 0.20 & 0.18 \\
\hline 95\% Confidence Interval & $1.90-2.05$ & $2.12-2.36$ \\
\hline Minimum value & 1.59 & 1.80 \\
\hline Maximum value & 2.36 & 2.52 \\
\hline
\end{tabular}

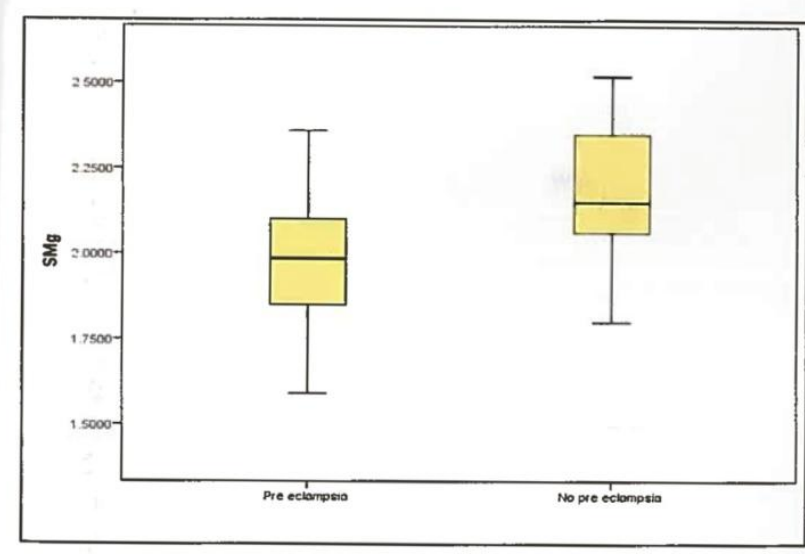

Chart 4. Box plot diagram Serum Magnesium in the study and control group. 
Hypomagnesemia was noted among preeclampsia subjects when compared to normal pregnant women.

\section{Discussion}

Pre-eclampsia is a pregnancy specific multi system disorder affecting $5-8 \%$ of pregnancies worldwide. Close observation and treatment with anti hypertensive agents and magnesium sulphate and if progressive signs and symptoms occur, urgent delivery of the fetus.

\section{Baseline Characteristics of the Study Population}

In the study population, a baseline characteristic like age distribution shows that the two groups are comparable with respect to age as shown in Table 1. Maximum study subjects are in the 20-24 and 25-29 age group in both the pre-eclampsia and no pre-eclampsia groups.

Most of the study subjects were in similar gestational groups. Both the groups were similar in gravidity. Majority of subjects from both the groups were below poverty line. Antenatal check up done by both groups.

\section{Analysis of Clinical Features of the Study Population}

The clinical feature like head ache oliguria epigastric pain foetal moments, dyspnoea were similar in both groups. None of the subjects are convulsion.

Table 1 \& Chart 1 shows the mean standard deviation of body mass index in the two groups. The mean value of mean and standard deviation of body mass index in the two groups. The mean value of BMI among pre-eclampsia patients was $28.9 \pm 3.3$ and that of normal pregnant women was $26.6 \pm 4 \%$. Chart 1 shows the box plot diagram of the distribution of subjects according to their Body Mass Index.

According to one study done by Shinako Yanamoto et al in 26 women with preeclampsia and 198 control women, where they recorded WHR, BW and BMI in early pregnancy, have found that WHR, BMI and BW early in pregnancy were significantly higher in the pre-eclampsia group $(\mathrm{p}<0.0001)$. In another systematic overview by Tara $\mathrm{O}$ Brien et al of thirteen cohort studies, have found that the risk of pre-eclampsia doubled with each $5-7 \mathrm{~kg} / \mathrm{m}$ increase in pre-pregnancy Body Mass Index. A total of 1,390,226 women were included in the 13 studies. ${ }^{13}$

In a study by Chanvitya Punthumapol et al in Thailand has found that in 30 normal pregnant women. In normal pregnant women, the BMI was $27.3 \pm 3.7$ and in preeclamptic women BMI was $30.2 \pm 4.3(\mathrm{p}<0.05){ }^{13}$

Table 2, Fig 2 A\&B

The mean value of systolic Blood Pressure among pre-eclampsia group was 147.3 with a SD of 12.1. Among the normal pregnant women the mean value was 113.4 with a SD of 8.2. The difference between the two means is statistically significant with a $\mathrm{p}$ value of 0.000 . The mean value of Diastolic Blood Pressure among pre-eclampsia group was 96.5 with a SD of 7.4. Among the normal pregnant women the mean value was 71.7 with a SD of 5.9. The difference between the two means is statistically significant with a $\mathrm{p}$ value of 0.000 .

Table 3 and Fig. 3 shows distribution of study subjects according to presence of urine protein, Out of the 30 pre-eclamptic women 29 had 1+ proteinuria with dipstick and only one had $3+$ proteinuria. Proteinuria is one of the criteria for diagnosing preeclampsia.

\section{Analysis of Serum Magnesium}

Tables 4 and Chart 4 shows the descriptive statistics of serum magnesium value in the study group. The mean serum magnesium value in preeclamptic women is $2 \pm 0.2$ and among normal pregnant women the mean is $2.2 \pm 0.2$. The obtained $\mathrm{p}$ value is significant when the difference between means was compared.

This is in accordance with the result found in the study by Adekanle et al in Nigeria in 2014 where they found lower serum magnesium levels in pre- 
eclamptic groups compared to normal pregnant women. $^{11}$

This is also in accordance with the result found in the study by Vahid Roodsari et al in Iran in $2007^{8}$ and by Nahar K et al in 2010 in Bangladesh. Similarly Sendhav Sandip et al in 2012 in Ahmedabad found low serum magnesium levels in severe preeclamptic patients. ${ }^{10}$

The hypomagnesemia in most pregnant women are associated with hemodilution due to estrogen, renal clearance during pregnancy and consumption of minerals by growing fetus. ${ }^{10}$ The consequences of low magnesium may lead to a reduction in cerebral blood flow, cerebral vasospasm and increase in neuronal burst. ${ }^{3}$

Hemodynamic abnormalities like arterial wall thickening, abnormal vascular tone and endothelial dysfunction due to alteration in the biology of cellular and non cellular components of arterial wall are caused by deficiency of magnesium. Magnesium is involved in blood pressure regulation by inhibiting NO synthase in endothelial cells. In hypomagnesemia production of ATP and ATP dependent sodium/potassium and calcium pump are impaired amplifying the pathogenesis. Magnesium deficiency during pregnancy has been associated with eclampsia, pre-eclampsia, preterm birth, increased maternal hospitalization, incidence of low birth weight and small for gestational age babies. ${ }^{12}$

Trials of oral magnesium supplementation during pregnancy have proved that there is reduction in hypertension, miscarriage, prenatal birth and fetal growth retardation. Treatment of convulsions has been helped by administration of magnesium sulphate. ${ }^{13}$

Response of the patient to parenteral magnesium sulphate may be due to the generalized sedative action and mild vasodilatory effect. ${ }^{119}$ Thus magnesium lowers blood pressure and alters peripheral vascular resistance and is advantageous for preeclamptic women. ${ }^{15}$

Analysis Of Ultarsonography Findings Among The Study Groups $30 \%$ of the pre-eclampsia group had IUGR whereas the normal group did not have IUGR. By applying Fishers exact test it was statistically significant.

In a study done in 200 SGA (Small for Gestational Age) babies in Peshawar, Pakistan by Taj Mohammed et al in 2010 have found that the risk of having an IUGR baby was 10 times higher for mothers who were primi para and had PIH.

In another study done among a total of 3010 mothers in Bhopal by Shweta Anand et al in 2011, it was found that LBW and SGA and preterm delivery was higher in PIH. ${ }^{14}$

\section{Summary and Conclusion}

Pre-eclampsia is a major cause of maternal and fetal morbidity and mortality especially in the developing nations. Risk factors are nulliparity, multi-fetal gestation, maternal age older than 25 years, increase in maternal weight. It is a two stage syndrome, initial stage being poor placentation and second stage is the maternal syndrome.

The study revealed the following features:

1. Mean BMI of pre eclamptic women were significantly higher than normal pregnant women.

2. Mean systolic and diastolic blood pressure among pre eclamptic women were significantly elevated than normal pregnant women.

3. Proteinuria was found among 29 preeclamptic women of the 30 protenuria was not seen in normal women.

4. The mean serum magnesium is significantly lower in pre-eclamphic patients.

5. Presents of IUGR was seen in $30 \%$ of pre eclamptic women.

It is highly recommendable to estimate serum magnesium of pregnant women. Low level of serum magnesium is modified by dietary modification with green leafy vegetables, fish, milk, almonds, legomes and pulses. Pharmacological interventions can be done under medical supervision in the form of magnesium tablets. 


\section{Relevance of the study}

- To find out serum magnesium level in the first trimester of pregnancy and correlation with prec-eclampsia.

- To supplement dietary magnesium and pharmacological intervention in the form of magnesium sulphate tables.

\section{Bibliography}

1. De Chermey AH, Nathan L, Goodwin TM, Laufer N. Current Diagnosis and Treatment - Obstetrics \& Gynaecology. Hypertension in pregnancy. $10^{\text {th }} \mathrm{Ed}$ New York, Me Graw Hill. 2007:318-326.

2. Park K. Park's Textbook of Preventive and Social Medicine $23^{\text {rd }}$ Ed. Jabalpur Banarsidas Bhanot. 2015: 558-560.

3. Zohreh T, Sara H. Comparison of maternal serum magnesium level in preeclampsia and normal pregnant women. Iran Re Cres Med J, December 2013; 15(12):e 10394.

4. Idogun ES, Imarengiaye CO, Momoh SM. Extra cellular calcium and L magnesium in Pre-eclampsia and Eclampsia. African J Reprod Health, 2007 August; 11(2): 89-94.

5. Indumati $\mathrm{V}$, Kodiwadmath $\mathrm{MV}$, Sheela MK. The role of serum electrolytes in cy induced hypertension. Journal of Clinical and Diagnostic Research, 2011 Feb; 5(1): 66-69

6. Chanvitya P. Boponsri K. Serum calcium, mangnesium and uric acid in pre eclampsia and normal pregnancy. J. Med.Assoc.Thai, 2008; 91(7)968-973.

7. Cunningham FG, Leveno KJ, Bloom SL, Hauth JC, Rose DJ, Spong CY. William's Obstetrics $23^{\text {rd }}$ Ed, New York: Me Graw Hill. 2010:706-752.

8. Hertig AT. Vascular pathology in the hypertensive albuminuric toxemias of pregnancy. Clinics 4, 1945:602.

9. Swaminathan R. Magnesium Metabolism and its disorders. Clin Biochem Rev, 2003;24: 47-66.
10. Fox C, Ramsoomair D, Carter C. Magnesium - its proven and potential clinical significance. South Med J, 2001; 94: 1195-1201.

11. Bandebuche S, Sagade B, Sontakke AN. Serum magnesium in pregnancy induced hypertension. Int J Med Sci, 2013 Nov; 1(4): 413-416.

12. Nahar K, Yasmin H, Shamsuddin L, Serum magnesium in pre-eclampsia and eclampsia. Bangladesh J Obstet and Gynaecol, 2010; 25(1): 15-19.

13. O'Brien TE, Ray JG, Chan W.S. Maternal Body Mass Index and the risk of preeclampsia: A systematic overview. Epidermiology, 2003; 14-368-374.

14. Makrides M, Crisvt DDm Bain E, Crowter CA ete al, magnesium supplementation in pregnancy. Cochrane database of sytematic reviews. 2014; 4: 1-73.

15. Pandhi P. Saha L. Malhotra S. Effect of oral magnesium supplementation on experimental pre-eclampsia induced by prolonged blockade of nitric oxide santhesis in pregnant rats. Indian Journal of Experimental Biology, 2002 march; 40: 349-351.

\section{List of Abbreviations:}

NO -Nitric Oxide

IUGR -Intra Uterine Growth Retardation

PIH -Pregnancy Induced Hypertension

SGA -Small for gestational Age

MMR -Maternal Mortality Rate 